Ann. Biol. anim. Bioch. Biophys., I969, 9 (4), 513-526.

\title{
EFFETS DES LÉSIONS DES NOYAUX VENTRO-MEDIANS HYPOTHALAMIQUES SUR LA PRISE D'ALIMENT CHEZ LE PORC
}

\author{
P. AUFFRAY \\ avec la collaboration technique de J.-C. Marcilloux et C. Daulouder \\ Station de Recherches sur l'Elevage des Porcs, \\ Centre national de Recherches zootechniques, 78 -Jouy-en-Josas \\ Institut national de la Recherche agronomique
}

\section{SOMMAIRE}

Sur des porcs de race Large White, pesant de 80 à $90 \mathrm{~kg}$, une lésion électrolytique bilatérale des noyaux ventro-médians de l'hypothalamus a été effectuée afin de déterminer dans cette espèce le rôle de ces noyaux dans le mécanisme de régulation alimentaire.

Dans ce but un appareil stéréotaxique, dérivé de celui d'Horsley-Clarke et adapté à la morphologie de l'animal, a été réalisé.

L'absence d'atlas stéréotaxique du cerveau du Porc et la diversité anatomique de ces animaux non sélectionnés et en cours de croissance, ont obligé de compléter la technique stéréotaxique classique par un repérage radiologique. Après iodo-ventriculographie, la structure à léser a été localisée par rapport à un point de référence (récessus tubérien du troisième ventricule).

La lésion bilatérale du noyau ventro-médian a déclenché une hyperphagie modérée mais nette, avec un accroissement de consommation atteignant $70 \mathrm{p}$. Ioo. Cette hyperphagie a toujours induit une obésité qui, après deux mois d'observation sur certains sujets était considérable et toujours croissante. Une différence entre sexes a été observée.

Ce résultat a été interprété comme une preuve de l'existence d'un mécanisme de régulation physiologique de la prise d'aliments chez le porc d'élevage et de l'intervention dans ce mécanisme des noyaux ventro-médians de l'hypothalamus.

\section{INTRODUCTION}

Depuis une trentaine d'années on connaît l'intervention des centres nerveux hypothalamiques sur la consommation des animaux. KELLER et al. (r935) ont montré la possibilité de produire une hyperphagie et de l'obésité en réalisant des destructions localisées au niveau de l'hypothalamus chez le Chien.

HETHERINGTON et RANSON (r940) ont constaté, chez le Rat, que les lésions les plus efficaces sont bilatérales et intéressent la région des noyaux ventro-médians. Ces résultats confirmés par plusieurs auteurs (BROBECK et al., I943 ; TEITELBAUM, 
I957) ont également été obtenus chez la Souris (MAYER et al., I955), le Chat (WHEATIEY, I944 ; ANAND et al., I955; KAELBER et al., I965), le Singe (BROOKs et al., I942; ANand et al., I955), et le Chien (HEINBECKER et al., I944); chez le Cobaye selon JOSEPH et al. (I968) de telles lésions ne donnent pas lieu à une hyperphagie.

Le Porc n'a fait l'objet d'aucune étude. Il nous a paru intéressant de pallier cette lacune compte tenu du comportement alimentaire particulier de cet animal qui 1'a fait considérer comme naturellement boulimique. Èn raison du poids élevé des animaux adultes, nous avons réalisé ce travail chez des porcs dont la croissance n'était pas terminée $(80-90 \mathrm{~kg})$.

\section{MATÉRIELS ET MÉTHODES}

Toute intervention stéréotaxique suppose la réalisation d'un appareillage mécanique.

Il n'existe pas d'atlas stéréotaxique du cerveau du Porc. En raison des variations individuelles importantes liées au fait que les animaux utilisés étaient en croissance et non sélectionnés sur des critères morphologiques, nous avons dû procéder au repérage des structures désirées par rapport à un système de référence.

\section{Appareillage stéréotaxique (fig. I)}

Pour atteindre les structures hypothalamiques impliquées dans la prise alimentaire, nous avons réalisé un cadre stéréotaxique dérivé de celui de Horsley et Clarke pour chat.

Description. - Les pièces permettant la fixation des barres d'oreilles sont de grande dimension, ce qui permet l'abaissement de la tête de l'animal et ainsi l'utilisation de l'appareillage stéréotaxique standard.

Chez le Porc, les conduits auditifs sont obliques et d'un accès difficile. Pour pallier cet inconvénient nous avons mis au point des barres d'oreilles dont l'extrémité est formée d'un cône faisant un angle déterminé avec la pièce qui lui est solidaire.

\section{Procédé de repérage anatomique}

A l'exemple des neuro-chirurgiens, nous choisissons des points de référence cérébraux au voisinage des formations anatomiques à localiser.

Nous situons les noyaux ventro-médians par rapport au récessus tubérien qui devient visible radiologiquement après introduction d'une substance de contraste (ici le lipiodol) dans le troisième ventricule (fig. 3 ).

L'appareil de radiologie utilisé, de type courant (puissance $80 \mathrm{kV}-28 \mathrm{~mA}$ ) est muni d'un dispositif de visée permettant le centrage des rayons $\mathrm{X}$ sur la région qui nous intéresse. En substituant un faisceau lumineux à la source de rayons $\mathrm{X}$ (dispositif A, fig. 2) on projette l'image d'un réticule sur un système de repérage. Ce dernier est monté sur un dispositif porte-drapeau à crémaillère qui peut glisser sur une des barres graduées du cadre.

Il comporte deux lames parallèles, l'une en plexiglass tournée vers la source de rayons $\mathrm{X}$, l'autre métallique à proximité de la zone à radiographier (dispositif $B$, fig. 2 ). Les rayons $X$ sont parfaitement centrés lorsqu'il y a coïncidence entre l'image de la figure géométrique donnée par la lame de plexiglass et celle tracée sur la lame métallique.

Les coordonnées stéréotaxiques des points $O$ ont été établies au préalable par rapport à l'axe des barres d'oreilles.

Un support cassettes monté sur un porte-électrode peut se déplacer sur l'autre barre du cadre stéréotaxique perpendiculairement à la direction des rayons $X$ (dimension $\mathrm{du} \mathrm{film} 9 / \mathrm{I} 2 \mathrm{~cm}$ ).

Sur une lame de plexiglass montée sur un dispositif porte-électrode est tracé un quadrillage dont les rainures remplies d'oxyde de plomb forment des carreaux de $2 \mathrm{~mm} / 2 \mathrm{~mm}$. Le centre de cette lame, percé d'un orifice contenant également de l'oxyde de plomb permet de faire le point zéro par rapport à l'extrémité des pointes d'oreilles. 
Ce quadrillage placé entre la préparation et le support cassettes permet sur le film radio. graphique le repérage de la structure cherchée en verticalité et en antériorité.

\section{Technique chirurgicale et conduite de l'intervention}

En raison du poids élevé des animaux $(80-90 \mathrm{~kg})$, de l'épaisseur de la voûte crânienne (environ $20 \mathrm{~mm}$ ), la peau est incisée et l'os aminci avant d'introduire dans le cadre la tête de l'animal. La craniectomie effectuée à l'aide d'un trépan de De Martel ne laisse subsister que quelques mm d'os au-dessus du cerveau.

Toute hémorragie osseuse est colmatée à l'aide de la cire à trépanation.

Pour des raisons anatomiques, la fixation de l'animal dans le cadre stéréotaxique se révèle généralement délicate : poids élevé, cou très court et puissant, conduits auditifs obliques et d'un accès difficile.

La première vérification d'un montage correct se fait à l'aide de l'extrémité des barres d'yeux, lesquelles doivent appuyer simultanément sur l'apophyse zygomatique.

Pour réaliser l'exploration radiologique, on descend l'extrémité d'un trocart de Clovis Vincent monté sur un porte-électrode, dans un des ventricules latéraux, quelques mm en avant du foramen de Monro à l'intersection de la perpendiculaire menée du sommet du trou optique avec l'os frontal. Ce point de repère est valable quels que soient le poids et la race de l'animal. Il est déterminé par une radiographie préalable, le trocart étant placé à l'extérieur de la tête du porc. Ensuite un orifice de quelques $\mathrm{mm}$ de diamètre à une latéralité de $\mathbf{L}=3 \mathrm{~mm}$ est pratiqué dans l'os à l'aide d'une fraise de dentiste. La dure-mère est alors percée à l'aide du biseau d'une aiguille hypodermique. Puis elle est incisée en croix au moyen de ciseaux fins après avoir pris soin de décoller les vaisseaux qui y adhèrent.

Après descente du trocart dans le ventricule choisi on réalise une radiographie de contrôle en incidence latérale. L'apparition de liquide céphalo-rachidien dans le trocart traduit un ton montage de la préparation. Ce liquide se déverse à l'extérieur au rythme des mouvements respiratoires. En cas d'insuccès, de l'os est enlevé a fin de découvrir le sinus longitudinal supérieur lequel permettra de corriger une erreur de latéralité.

Après extraction de un à deux $\mathrm{ml}$ de liquide céphalo-rachidien, un $\mathrm{ml}$ de lipiodol (substance de contraste) est injecté lentement ( 4 à 5 secondes) dans la corne antérieure du ventricule latéral. Cette substance passe alors dans le troisième ventricule et réalise ainsi son opacification. Quelques minutes après l'injection du contraste, on réalise une radiographie.

Le lipiodol quitte rapidement le troisième ventricule pour s'engager dans l'aqueduc de Sylvius. Néanmoins, pendant la durée des manipulations, il en persiste suffisamment dans la portion du troisième ventricule sous-jacente au niveau de l'aqueduc pour opacifier cette zone.
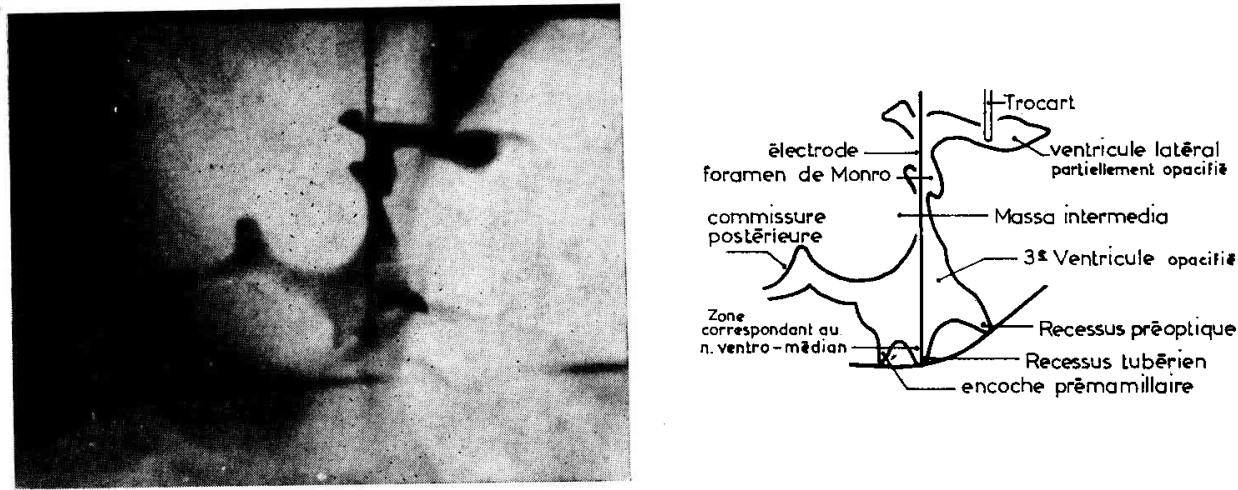

FIG. 3. - Repérage anatomo-radiologique des royaux ventro-médians hypothalamiques

Pour localiser les noyaux ventro-médians hypothalamiques nous avons effectué chez 8 porcs de nombreuses coupes histologiques en cherchant à déterminer les traces laissées par les électrodes au niveau du tissu nerveux (dans la fig. 3 la pointe de l'électrode verticale se trouve an niveau de l'infundibulum tubérien). 
Ces noyaux sont faciles à identifi $r$ en raison de leur aspect ovale avec unc concentration cellulaire plus dense à la périphárie.
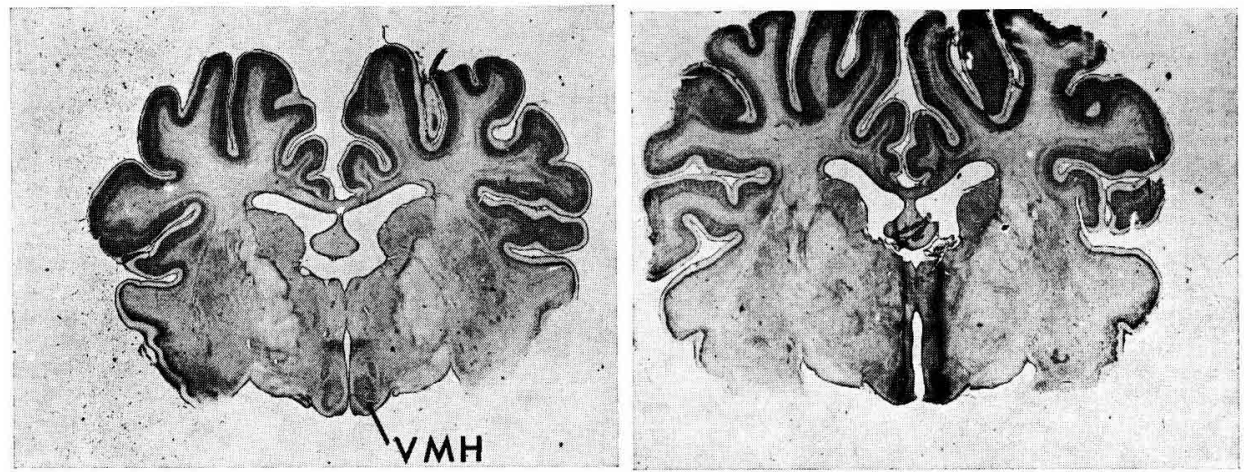

FIG. 4. - Coupes frontales du cerveau en vraie grandeur intéressant les noyaux ventro-médians A droite on peut voir les traces laissées par les électrodes aboutissant à ces noyaux (V. M. H. = noyau ventro-médian hypothalamique)

\section{PROTOCOLE EXPÉRIMENTAL,}

L'ensemble des recherches qui sont exposées ici ont été effectuées sur des porcs de race Large-White d'un poids compris entre 80 et $90 \mathrm{~kg}$.

Après un jeûne de 36 heures les animaux sont soumis à une anesthésie très profonde. Celle-ci consiste en une injection intraveineuse d'un mélange de Nembutal, de Penthiobarbital et d'atropine, dans les proportions qui suivent:

\begin{tabular}{|c|c|}
\hline Nembutal & $5 \mathrm{ml}$ (solution à 6,5 p. IOO) \\
\hline Penthiobarbital $\ldots \ldots \ldots \ldots$ & $\begin{array}{l}\mathrm{I} \mathrm{g} \\
2 \mathrm{ml} \text { (solution }\end{array}$ \\
\hline $\begin{array}{l}\text { Atropine (sulfate) } \ldots \ldots \ldots \ldots \ldots \ldots \\
\text { Eau distillée q. s. p. } \ldots \ldots \ldots \ldots \ldots\end{array}$ & $2 \mathrm{ml}$ (solution à $2 \mathrm{p} . \mathrm{IOO})$ \\
\hline
\end{tabular}

Après intubation trachéale, l'anesthésie est entretenue à l'aide d'un mélange gazeux (oxygène-protoxyde d'azote), ce mélange pouvant ou non barboter dans du méthoxyfluorane (penthrane) suivant le degré d'anesthésie souhaitée.

Les lésions ont été réalisées à l'aide d'électrodes d'acier de diamètre $0,7 \mathrm{~mm}$ et recouvertes d'un vernis sauf au niveau de la partie affinée qui a une longueur de I mm. On fait passer dans ces électrodes un courant de $5 \mathrm{~mA}$ pendant $30 \mathrm{~s}$. L'électrode indifférente est fixée au niveau de la commissure des lèvres. La descente de l'électrode de coagulation se fait selon les techniques classiques; dans notre cas à une latéralité $\mathrm{L}_{\mathrm{L}}=2 \mathrm{~mm}$, au niveau de l'encoche tubérienne.

Chez les animaux témoins nous ne réalisons que la ventriculographie.

Les animaux nourris ad libitum reçoivent un régime dont la composition est la suivante :

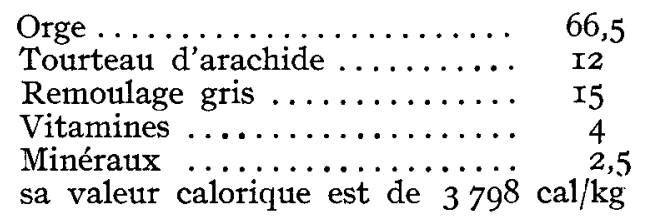


Nous mesurons la consommation journalière et les animaux sont pesés une fois par semaine.

Après une pétiode d'observation de huit semaines, les animaux sont sacrifiés. Quelques animaux ont été conservés pendant trois mois. Après abattage de l'animal, le cerveau est perfusé en introduisant dans les carotides une solution de ferrocyanure de potassium à I p. Ioo (mise en évidence đu dépôt métallique réalisé lors de l'électrolyse) suivie d'une solution de formol à Io p. Ioo. Le cerveau est laissé en place trois jours dans la boîte crânienne plongée dans le formol pour éviter les déformations. Puis la fixation complète du cerveau extirfé est poursuivie dans le formol pendant une quinzaine de jours.

Les coupes histologiques sont réalisées à congélation (épaisseur Ioo $\mu$ ) et colorées à la thionine (NISSL).

\section{RÉSULTATS}

Les résultats suivants sont tirés de l'observation de 22 porcs de race Large White. Ils concernent à la fois des mâles castrés et des femelles d'un poids compris entre 80 et $90 \mathrm{~kg}$. Les différentes données sont reproduites dans les figures 5 à 8 et les tableaux I à 4 .

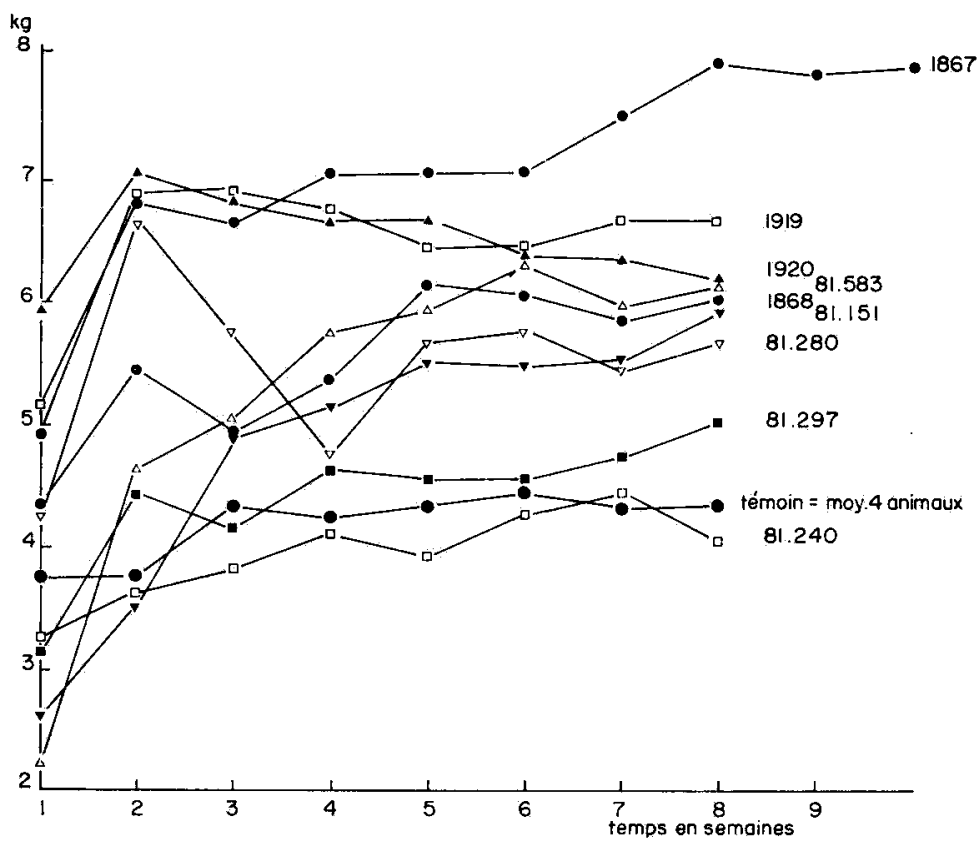

FIG. 5. - Ingestion quotialienne des porcs males castrés

(en ordonnée les quantités consommées en $\mathrm{kg}$, en abscisse le temps en semaines)

Le Porc no 81240 est non hyperphagique mais obèse. La chute de consommation chez le porc $n^{\circ} 81280$ est due à une atteinte de broncho-pneumonie 


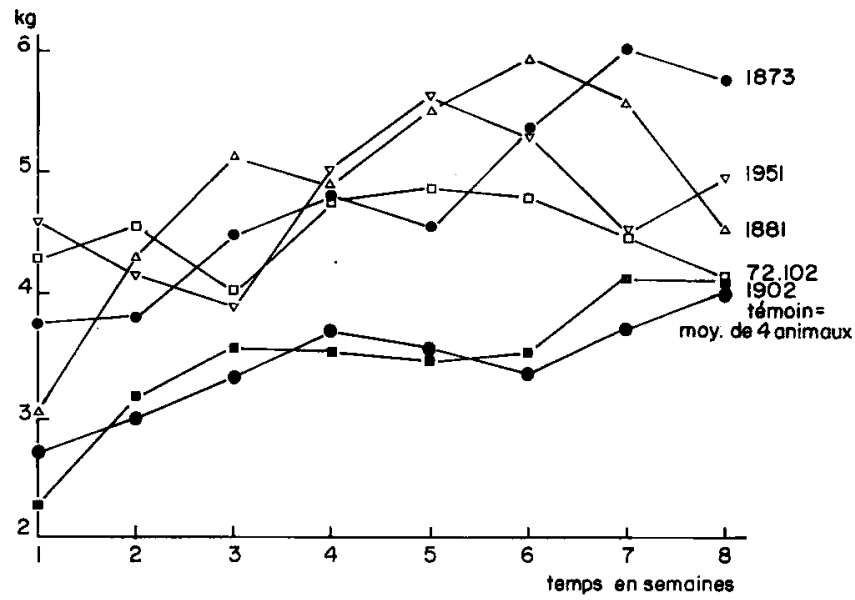

FIG. 6. - Ingestion quotidienne de porcs femelles

(en ordonnée les quantités ingérées en $\mathrm{kg}$, en abscisse le temps en semaines)

Le porc $n^{\circ}$ I95 I est hyperphagique et polydipsique

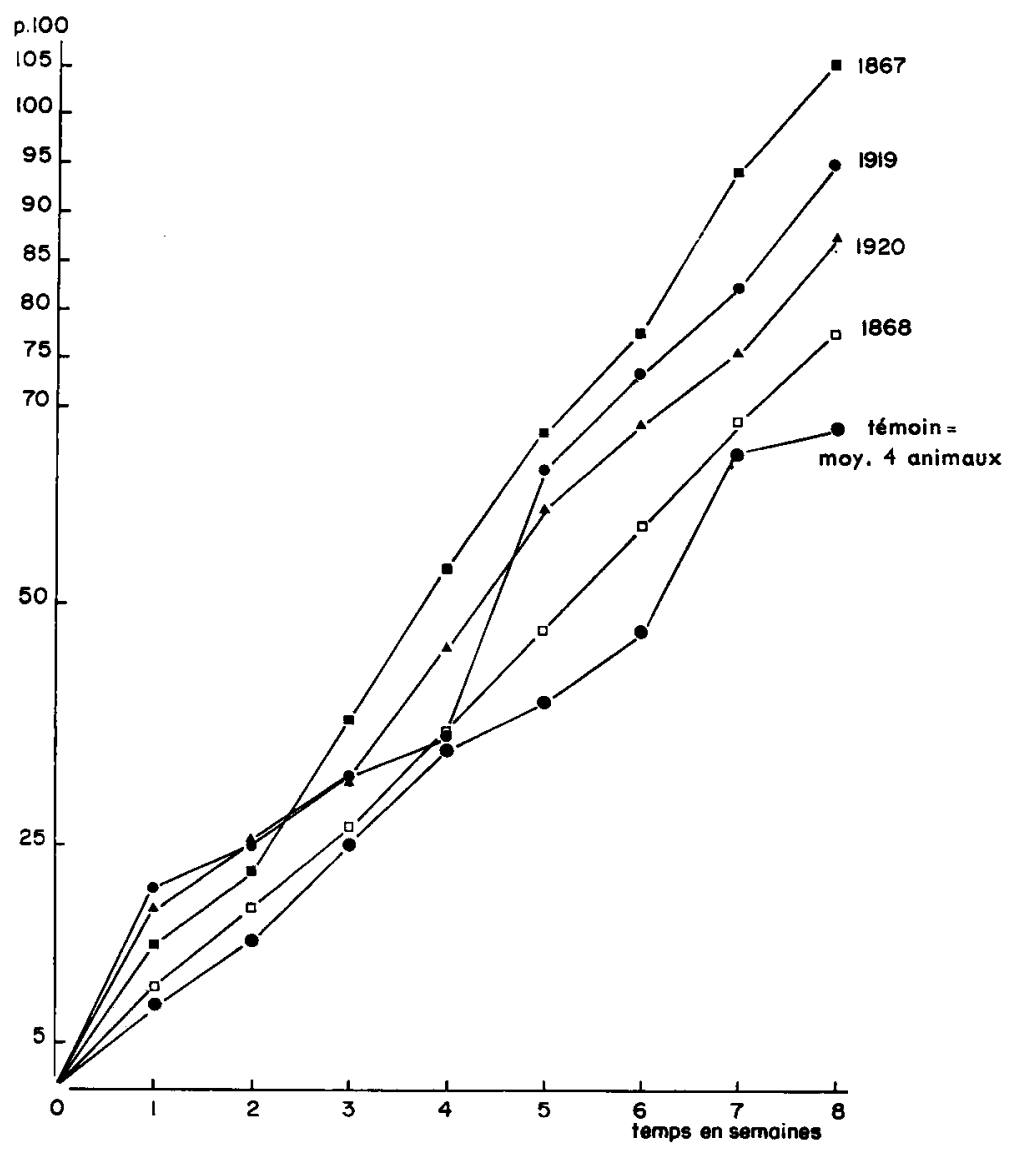

FıG. 7. - Gain đade poids de mâles castrés en p. 100 du poids initia! 


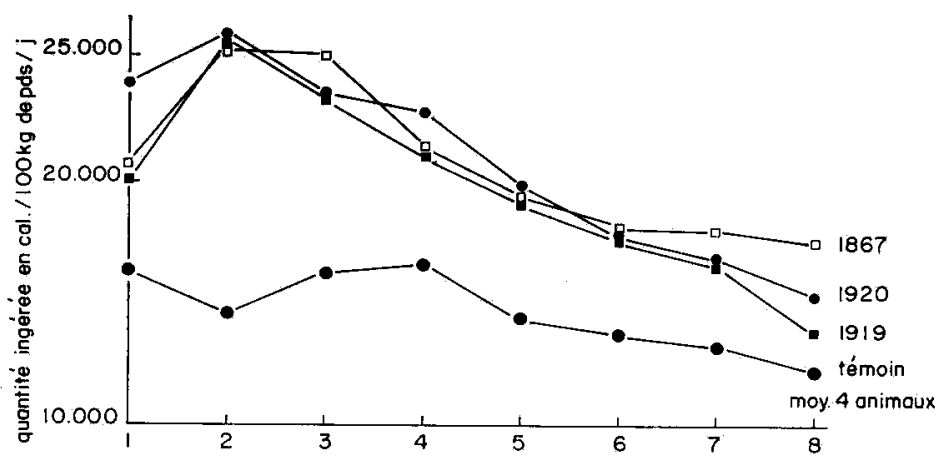

Fig. 8. - Quantités ingérées en cal/100 kg de poids/jour chez des mâles castrés

\section{TABLEAU I}

Ingestion quotidienne d'aliment en $k g$ chez les mâles castrés "coagulés" et estimation des lésions médio-ventrales hypothalamiques

\begin{tabular}{|c|c|c|c|c|c|c|c|c|c|c|}
\hline \multirow[t]{2}{*}{ Porc $n^{0}$} & \multicolumn{8}{|c|}{ Temps en semaines } & \multicolumn{2}{|c|}{$\begin{array}{l}\text { P. } 100 \text { approximatif } \\
\text { de destruction } \\
\text { des V.M. H. }\end{array}$} \\
\hline & 1 & $\underline{9}$ & 3 & 4 & 5 & 6 & 7 & 8 & Gauche & Droit \\
\hline 81240 & 3,2 & 3,6 & 3,8 & 4,1 & 3,9 & 4,3 & 4,4 & 4,0 & 0 & 0 \\
\hline $1956 \ldots$ & 2,2 & 3,9 & 3,6 & 3,7 & 4,4 & 4,6 & 4,7 & 4,7 & 0 & 0 \\
\hline $81297 \ldots$ & 3,1 & 4,4 & 4,1 & 4,6 & 4,5 & 4,5 & 4,7 & 5,0 & 0 & 0 \\
\hline $81280 \ldots$ & 4,2 & 6,6 & 5,7 & 4,6 & 5,8 & 5,9 & 5,4 & 5,6 & 80 & 70 \\
\hline $81151 \ldots$ & 2,5 & 3,7 & 4,9 & 5,1 & 5,7 & 5,5 & 5,7 & 5,9 & 100 & 25 \\
\hline $1868 \ldots$ & 4,3 & 5,4 & 4,9 & 5,5 & 6,1 & 6,0 & 5,8 & 6,0 & 60 & 80 \\
\hline $81583 \ldots$ & 2,1 & 4,6 & 5,0 & 5,5 & 5,9 & 6,3 & 5,9 & 6,1 & latérale & latérale \\
\hline 1920 & 5,9 & 7,0 & 6,8 & 6,6 & 6,6 & 6,3 & 6,3 & 6,1 & 95 & 85 \\
\hline 1919 & 4,9 & 6,8 & 6,9 & 6,7 & 6,4 & 6,4 & 6,6 & 6,6 & 80 & 100 \\
\hline 1867 & 5,1 & 6,8 & 6,6 & 7,0 & 7,0 & 7,0 & 7,5 & 7,9 & 100 & 100 \\
\hline
\end{tabular}

Ingestion quotidienne d'aliment en $\mathrm{kg}$ chez les mâles castrés témoins

\begin{tabular}{|c|c|c|c|c|c|c|c|c|}
\hline \multirow{2}{*}{ Porc no } & \multicolumn{8}{|c|}{ Temps en semaines } \\
\hline & 1 & 2 & 3 & 4 & 5 & 6 & 7 & 8 \\
\hline $1929 \ldots \ldots$ & 3,6 & 3,6 & 4,9 & 4,2 & 4,1 & 4,1 & 4 & 4 \\
\hline $81314 \ldots \ldots \ldots$ & 4,9 & 5 & 5,3 & 5,6 & 5,2 & 5,5 & 5,3 & 5,1 \\
\hline $72089 \ldots \ldots \ldots$ & 3,9 & 3,4 & 4,2 & 3,5 & 4,4 & 4,3 & 4,7 & 4,6 \\
\hline $81138 \ldots \ldots \ldots$ & 2,7 & 2,3 & 2,9 & 3,5 & 3,5 & 3,9 & 3,5 & 3,6 \\
\hline Moyenne..... & 3,7 & 3,7 & 4,3 & 4,2 & 4,3 & 4,4 & 4,3 & 4,3 \\
\hline
\end{tabular}


TABLEAU 2

Gain de poids quotidien en kg des mâles castrés et "coagulés"

\begin{tabular}{|c|c|c|c|c|c|c|c|c|}
\hline \multirow{2}{*}{ Porc $n^{\circ}$} & \multicolumn{8}{|c|}{ Temps en semaines } \\
\hline & 1 & 2 & 3 & 4 & 5 & 6 & 7 & 8 \\
\hline $81240 \ldots \ldots \ldots$ & 0,1 & 0,6 & 0,2 & 0,6 & 0,7 & 1 & 0,4 & 0,9 \\
\hline $1956 \ldots \ldots \ldots$ & 0,4 & 1 & 0,9 & 0,9 & 1,4 & 0,6 & 1 & 1,1 \\
\hline $81297 \ldots$ & 0,4 & 0,7 & 1,9 & 0,7 & 1 & 1 & 0,6 & 1 \\
\hline $81280 \ldots$ & 1,7 & 1,3 & 0,3 & 1 & 1,1 & 1,1 & 1,1 & 0,0 \\
\hline $81151 \ldots \ldots \ldots$ & 0,6 & 1,3 & 1,6 & 1 & 1 & 1,1 & 1 & 1,1 \\
\hline $1868 \ldots \ldots \ldots$ & 0,6 & 0,9 & 0,9 & 1,1 & 1,3 & 1,1 & 1,3 & 1 \\
\hline $81153 \ldots \ldots \ldots$ & 0,2 & 0,7 & 1,3 & 1,4 & 1,7 & 0,6 & 1,3 & 1,'́t \\
\hline $1919 \ldots$ & 1,3 & 1,1 & 1,7 & 0,9 & 1,3 & 1,7 & 1,3 & $1, ' k$ \\
\hline $1920 \ldots$ & 1,3 & 1,4 & 0,6 & 1,4 & 1,1 & 1,7 & 1 & 1 \\
\hline $1867 \ldots$ & 1,9 & 0,6 & 1,9 & 2 & 2 & 1,3 & 2 & 1,4 \\
\hline
\end{tabular}

Gain de poids quotidien en $\mathrm{kg}$ chez les mâles castrés témoins

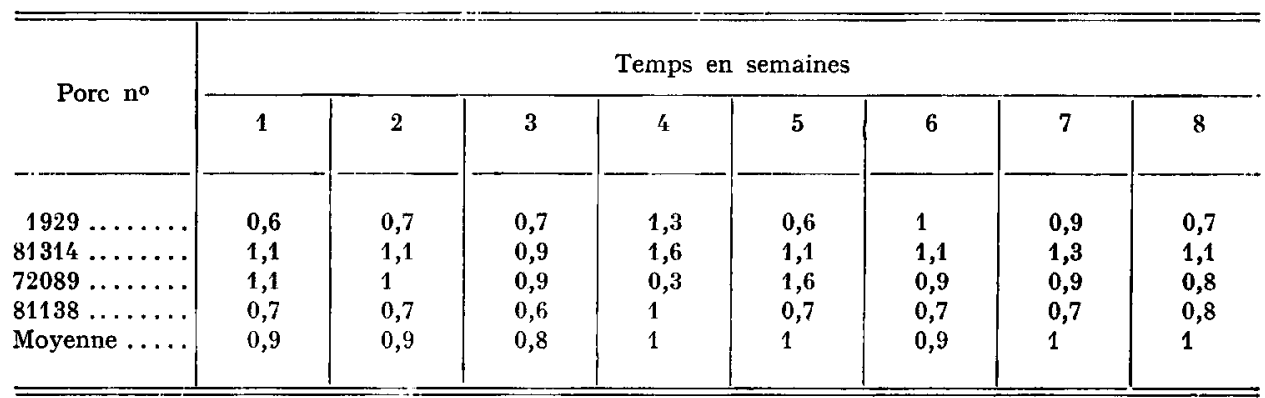

TABLEAU 3

Ingestion quotidienne d'aliment en hg chez les femelles "coagulées" et estimation des lésions médio-ventrales hypothalamiques

\begin{tabular}{|c|c|c|c|c|c|c|c|c|c|c|}
\hline \multirow[t]{2}{*}{ Porc $\mathrm{n}^{\circ}$} & \multicolumn{8}{|c|}{ Temps en semaines } & \multicolumn{2}{|c|}{$\begin{array}{l}\text { P. } 100 \text { approximatif } \\
\text { de destruction } \\
\text { des V.M. H. }\end{array}$} \\
\hline & 1 & 2 & 3 & 4 & 5 & 6 & 7 & 8 & Gauche & Droit \\
\hline $1873 \ldots$ & 3,8 & 3,6 & 4,5 & 4,8 & 4,6 & 5,4 & 6 & 5,8 & 60 & 90 \\
\hline $1951 \ldots$ & 4,6 & 4,2 & 3,9 & 5 & 5,6 & 5,3 & 4,5 & 5 & 100 & 100 \\
\hline $1871 \ldots$ & 2,3 & 4,3 & 5 & 4,9 & 5,5 & 6 & 5,6 & 4,3 & 70 & 80 \\
\hline $72102 \ldots$ & 4,3 & 4,6 & 4 & 4,8 & 4,8 & 4,8 & 4,2 & 4,2 & 0 & 100 \\
\hline $1902 \ldots$ & 2,2 & 3,7 & 3,6 & 3,5 & 3,5 & 3,5 & 4,1 & 4 & 0 & 0 \\
\hline
\end{tabular}




\section{TABLEAU 3 (suite)}

Ingestion quotidienne d'aliment en $\mathrm{kg}$ chez les femelles témoins

\begin{tabular}{|c|c|c|c|c|c|c|c|c|}
\hline \multirow{2}{*}{ Porc $n^{\circ}$} & \multicolumn{8}{|c|}{ Temps en semaines } \\
\hline & 1 & 2 & 3 & 4 & 5 & 6 & 7 & 8 \\
\hline $1906 \ldots$ & 3 & 3 & 4 & 3,7 & 3,2 & 3,6 & $l_{4}$ & 3,8 \\
\hline $1955 \ldots \ldots$ & 2,7 & 2,6 & 2,4 & 3,9 & 4 & 2,4 & 3,3 & 3,0 \\
\hline $1780 \ldots \ldots$ & 2 & 3,3 & 3,3 & 3,6 & $3, \mathbf{k}$ & 3,5 & 3,9 & 3,6 \\
\hline $1901 \ldots$ & 2,9 & 3,2 & 3,6 & 3,4 & 3,4 & 4 & 3,7 & 4,6 \\
\hline Moyenne .... & 2,6 & 3 & 3,3 & 3,6 & 3,5 & 3,2 & 3,7 & 3,9 \\
\hline
\end{tabular}

TABLEAU 4

Gain de poids quotidien en kg chez les femelles "coagulées"

\begin{tabular}{|c|c|c|c|c|c|c|c|c|}
\hline \multirow{2}{*}{ Porc $n^{\circ}$} & \multicolumn{8}{|c|}{ Temps en semaines } \\
\hline & 1 & 2 & 3 & 4 & 5 & 6 & 7 & 8 \\
\hline 1873. & 1,1 & 0,6 & 0,7 & 0,9 & 0,9 & 0,9 & 1,3 & 1,3 \\
\hline $1951 \ldots \ldots$ & 1,1 & 0,6 & 0,5 & 1,1 & 1,6 & 0,9 & 0,3 & 1,1 \\
\hline $1871 \ldots \ldots$ & 0,7 & 1,3 & 1,4 & $1,{ }^{\prime}$ & 0,9 & 1,3 & 1,3 & 0,6 \\
\hline $72102 \ldots \ldots$ & 1,4 & 0,6 & 1 & 1,1 & 1,1 & 0,7 & 0,7 & 1,1 \\
\hline $1902 \ldots$ & 1,1 & 0,6 & 0,9 & 0,9 & 0,7 & 1 & 0,8 & $0,{ }_{1}^{\prime}$ \\
\hline
\end{tabular}

Gain de poids quotidien en $\mathrm{kg}$ chez les femelles témoins

\begin{tabular}{|c|c|c|c|c|c|c|c|c|}
\hline \multirow{2}{*}{ Porc $n^{\circ}$} & \multicolumn{8}{|c|}{ Temps en semaines } \\
\hline & 1 & 2 & 3 & 4 & 5 & 6 & 7 & 8 \\
\hline 1906. & 0,6 & 0,6 & 1,3 & 0,3 & 0,1 & 1,7 & 0,9 & 0,4 \\
\hline $1955 \ldots \ldots \ldots$ & 1 & 0,6 & 0,6 & 1,1 & 0,9 & 1,3 & 1 & 0,9 \\
\hline $1785 \ldots \ldots \ldots$ & 1 & 0,9 & 0,9 & 1,3 & 0,5 & 1 & 0,9 & 1 \\
\hline $1901 \ldots \ldots \ldots$ & 1,1 & 1,3 & 1 & 0,6 & 0,9 & 1 & 0,7 & 1,3 \\
\hline Moyenne .... & 0,9 & 0,8 & 0,9 & 0,8 & 0,6 & 1,2 & 0,9 & 0,9 \\
\hline
\end{tabular}

1. Infuence des lésions médio-ventrales hypothalamiques sur la consommation et le gain de poids

Comparés aux animaux témoins, les mâles castrés coagulés consomment davantage. Cela se manifeste dès la première semaine post-opératoire. Les semaines suivantes cet accroissement de l'ingestion s'accentue. Cependant la consommation rapportée au poids de l'animal diminue généralement un mois après l'opération sans 
atteindre celle des témøins (fig. 8). Cette prise d'aliment peut être dans les cas favorables assimilée à une véritable hyperphagie (elle peut en effet dépasser de 70 p. Ioo celle des témoins).

Les femelles ayant subi des lésions ventro-médianes mangent également davantage, toutefois dans des proportions moindres que les mâles castrés. De plus, cet accroissement de 1'ingestion revêt un aspect irrégulier.

Les différences dans les réponses sont liées à l'importance des lésions et à leur localisation. Les réponses les plus efficaces proviennent des lésions des noyaux ventromédians. Des destructions immédiatement latérales à ces noyaux et, dans le même plan rostro-caudal, donnent lieu à une augmentation de la consommation (porc $n^{\circ} 85^{8} 83$ ). Les lésions les plus efficaces sont bilatérales intéressant les deux centres, mais nous obtenons une réponse très nette après coagulation d'un seul noyau ventro-

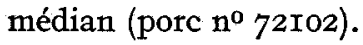

A une augmentation de consommation chez les mâles castrés correspond un accroissement pondéral proportionnel. Par contre, le gain de poids des femelles ayant des lésions ventro-médianes ne semble pas présenter de différence significative avec celui des témoins.

Ajoutons que le porc mâle $n^{\circ} \mathrm{I} 867$, conservé pendant trois mois, a doublé son poids au cours des sept semaines post-opératoires et triplé celui-ci au bout de trois mois tout en maintenant son niveau d'ingestion constant $(8-8,5 \mathrm{~kg}$ par jour).

Chez un porc (n० I95I) nous avons observé simultanément de 1'hyperphagie et de la polydipsie : 45-47 litres d'eau consommés par jour (normalement IO-I 5 litres). Les destructions intéressent à la fois les noyaux ventro-médians et la base du cerveau.

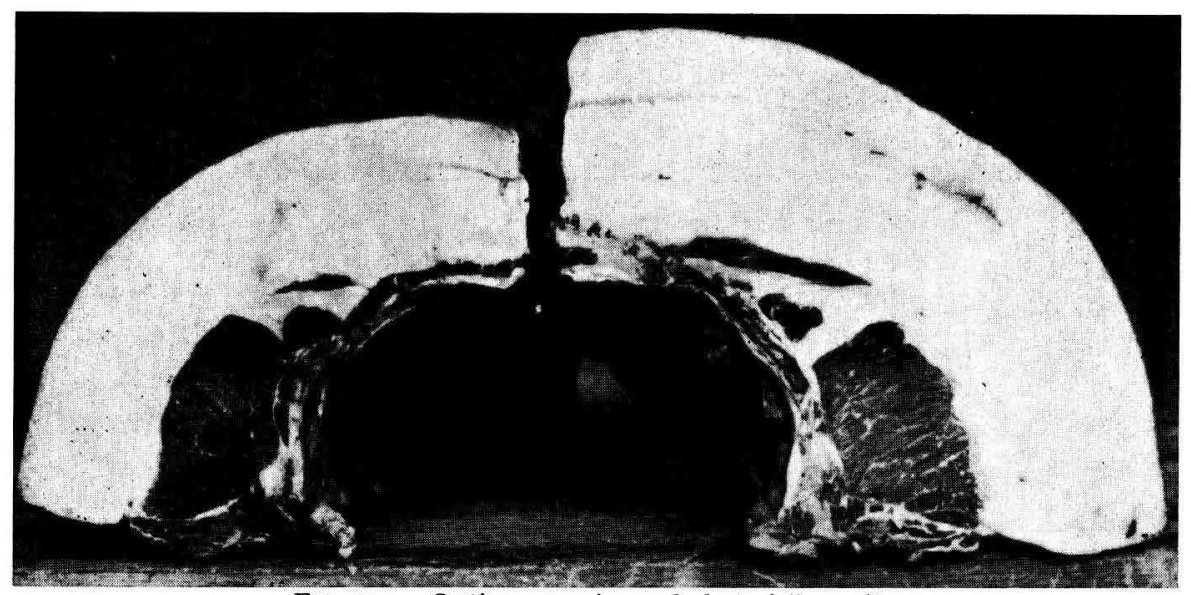

FIG. 9. - Seciions au niveau àe la ireizième côte

à gauche : animal témoin à droite : animal coagulé

Cette surconsommation quand elle existe, entraîne de l'obésité. Il y a envahissement de tout l'organisme par les graisses. Apparemment la croissance musculaire ne semble pas altérée. Cependant les muscles présentent une infiltration graisseuse nette (fig. 9). 


\section{Comportement des animaux opérés}

Les porcs ayant subi des lésions électrolytiques des noyaux ventro-médians deviennent apathiques.

Nous ne savons pas si les animaux traités et nourris ad libitum modifient leur rythme d'alimentation au cours du nycthémère. En effet les porcs en expérience étaient placés à proximité d'autres animaux recevant trois repas par jour.

Nous n'avons jamais observé une excitation au cours de la phase post-opératoire, excitation qui a été souvent décrite chez les autres espèces étudiées.

\section{DISCUSSION}

A la suite des expériences de KEnNEDy (I950) ; de HAN et al. (I965) ; de BERNARDIs et al. (I956), on sait que les très jeunes rats n'augmentent pas leur consommation après des lésions médio-ventrales. L'ingestion, rapportée au poids corporel les fait considérer comme des hyperphagiques physiologiques. L'effet de ces destructions hypothalamiques devient net lorsque l'animal se rapproche de l'état adulte. Quand la croissance est achevée la réponse est maximum. En ce qui concerne le Porc, on constate un accroissement important de la prise d'aliment qui dans les cas favorables peut être assimilé à une hyperphagie. Cela se produit bien que la croissance de cet animal soit loin d'être terminée. On ne peut en raison de leur poids, utiliser des animaux adultes (quelques centaines de $\mathrm{kg}$ ). Une telle expérience serait possible avec des porcs miniatures (cent $\mathrm{kg}$ au maximum en fin de croissance).

L'évolution de 1'hyperphagie chez le Rat se fait selon deux phases :

- une phase dynamique pendant laquelle l'animal double sa consommation et devient obèse ;

- une phase statique au cours de laquelle 1'ingestion de nourriture par les animaux coagulés devient similaire à celle des témoins.

En ce qui concerne les porcs ayant des lésions médio-ventrales nous constatons une diminution dans la consommation au fur et à mesure du déroulement de l'expérience mais, elle reste toujours supérieure à celle des témoins. Peut-être cela est-i1 dû à une période expérimentale trop courte (8 semaines) ou à l'utilisation d'animaux en croissance ?

L'intensité de la réponse est liée à l'importance de la destruction des noyaux ventro-médians hypothalamiques. Mais une lésion unilatérale peut donner lieu à un accroissement d'ingestion. Cela avait déjà été signalé par MAYER et BARNETTT (I955).

Des réponses peuvent être obtenues après destructions des structures immédiatement adjacentes aux structures médio-ventrales. Selon ANAND et BROBEcK (I95I) il $\mathrm{y}$ aurait interruption des voies nerveuses entre l'aire hypothalamique latérale considérée comme " un centre de la faim " (feeding center) et le noyau ventro-médian défini comme un " centre de la satiété " (satiety center) ou centre inhibiteur de cette aire latérale.

Des auteurs détruisant les structures ventro-médianes, surtout chez le Rat et le Chat ont transformé des animaux bien apprivoisés en animaux sauvages. Ce chan- 
gement comportemental va souvent de pair avec l'hyperphagie et l'obésité. De telles réactions, observées même chez de jeunes animaux, n'ont jamais été constatées par nous chez le Porc.

En résumé, il est possible d'utiliser un appareil stéréotaxique dérivé de celui de Horlsey et Clarke pour Chat chez le Porc jusqu'à un poids d'une centaine de kg.

La variabilité de la forme du crâne qui résulte de l'absence de sélection de ce caractère, ainsi que l'utilisation d'animaux en croissance nous ont conduit à situer les structures ventro-médianes par rapport à un point de référence cérébral : l'infundibulım tubérien après opacification du troisième ventricule. Ce mode de repérage indispensable permet d'atteindre les structures nerveuses désirées dans environ 80 p. Ioo des cas.

Après destruction des noyaux ventro-médians, les animaux mangent davantage et, dans les cas favorables ils peuvent être considérés comme hyperphagiques. Les effets dépendent non seulement du lieu, mais aussi de l'importance des destructions. Toute surconsommation conduit à de l'obésité.

A la suite de ces lésions les porcs deviennent apathiques.

On n'observe jamais une phase d'excitation consécutive à ces lésions.

Reçu pour publication en avril 1969.

\title{
REMERCIEMENTS
}

Nous remercions MM. BARBREL et Fouché pour la réalisation du cadre stéréotaxique et MM. BONNET et VerGe pour la mise au point de l'appareillage destiné au centrage des rayons $\mathrm{X}$.

\section{SUMMARY}

\author{
EFFECT OF VENTROMEDIAI HYPOTHALAMIC LESIONS
} ON FOOD INTAKE IN THE PIG

The effect of ventromedial hypothalamic lesions on food intake was studied on Large White pigs of 80 to $90 \mathrm{~kg}$ of weight. Female and castrated male animals received bilateral electrolytic lesions placed with a modified HoRSLEY and CLARKE's stereotaxic instrument adapted to the conformation of the pig (fig. I). The lack of stereotaxic atlas for the pig brain and the variations of anatomical characteristics between pigs (growing, non-selected animals), compelled us to useX-ray localization under ventricle lipiodol injection in addition to the stereotaxic technique. The position of the VMH nuclei was deterpined using the tuberal recess of the third ventricle as a point of reference (fig. 2 et 3 ).

The bilateral lesion of VMH nuclei induced a 70 per cent increase in food intake. This a hyperphagia " reached its maximum one week after the lesion. It was more marked in the male (fig. 5 et 6 ) than in the female in which it appeared more irregular (fig. 6).

No dynamic followed by a static phase was observed ; food intake remained at a high rate during the 8 weeks of the experiment, possibly due to the fact that growing animals were used for a comparatively short period.

Considerable and still increasing obesity was observed after 2 months (fig. 9), with a difference between sexes.

After lesion, the pigs became listless and no subsequent excitation phase was noticed.

Our results give evidence for the presence of a physiological mechanism regulating food intake in the pig, and for the part played in it by the VMH nuclei. 


\section{RÉFÉRENCES BIBLIOGRAPHIQUES}

ANAND B. K., Brobeck J. R., 195I. Localization of a feeding center " in the hypothalamus of the rat. Proc. Soc. Exp. Biol, Med., 77, 323-324.

ANAND B. K., Brobeck J. P., I95I. Hypothalamic control of food intake in rats and cats. Yale. J. Biol. Med., 24, 123-140.

Anand B. K., Dua S., Shoenberg K., I955. Hypothalamic control of food intake in cats and monkeys. J. Physiol. London, 12\%, r43-152.

BERNARDIS L. L., I966. Development of hyperphagia in female rats with ventromedial hypothalamic lesions placed at four different ages. Experientia, 22, 593-596.

Brobeck J. R., Tepperman J., Long C. N. H., 1943. Experimental hypothalamic hyperphagia in the albino rat. Yale J. Biol. Med., 15, 83I-833.

Brooks C., Mc. C., Lambert E. F., Bard P., 1942. Experimental production of obesity in the monkey. Federat. Proc., 1, II.

HAN P. W., IND C. H., CHU C., MU J., LIU A.C., I965. Hypothalamic obesity in Weanling rats. Am. J. Physiol., 209, 627-63r.

Hetherington A. W., Ranson S. W., r940. Hypothalamic lesions and adiposity in the rat. Anat. Record, 78, I49-172.

Heinbecker P., White H. L., Doris R., I944. Experimental obesity in the dog. Am. J. Physiol., 141, 549-565.

JoSEPH S. A., KNIGGE K. M., 1968. Effects of V. M. H. lesions in adult and newborn Guinea pigs. Neuroendocrinology, 3, 309-33I.

Keller A. D., Noble W., 1935. Adiposity with normal sex function following extirpation of the posterior lobe of hypophysis in the dog. Am. Physiol. Soc., 113, 79-80.

Kennedy G. C., 1950. The hypothalamic control of food intake in rats. Proc. Roy. Soc. London, Ser. B., $137,535-549$.

Mayer J., French R. G., Zighira C. F., Barnett R. J., I955. Hypothalamic obesity in the mouse: production, description and metabolic characteristics. Am. J. Physiol., 182, 75-82.

Teitelbaum P., I957. Random and food directed activity in hyperphagic and normal rats. J. Comp. Physiol. Psychol., 50, 486-49o.

Wheatley M. D., I944. The hypothalamus and affective behavior in cats. A study of the effects of experimental lesions with anatomic correlations. Arch. Neurol. Psychiat., 52, 296-316. 